\title{
Doktor $(\mathfrak{C} \mathfrak{a} \mathfrak{d} \mathfrak{i} \mathfrak{i s}$
}

(Fine wahre (s)efdidte

opn

F. Marion Cramioro.

Autorifirte Heberjetung

Doit

Iq́. \$öpfuer.

\section{$\mathfrak{B} \in \mathfrak{r} \mathfrak{l} \mathfrak{i}$,}

Drữ und Berlag von Seorg Reimer.

1892. 
